\title{
Role of nitric oxide in septal coronary arteries of obese Zucker rats
}

\author{
Priyanka Prathipati ${ }^{1}$, Syed Quadri ${ }^{1}$, Debra W. Jackson ${ }^{2}$, Keith E. Jackson ${ }^{1 *}$ \\ ${ }^{I}$ Department of Basic Pharmaceutical Sciences, College of Pharmacy, University of Louisiana at Monroe, \\ 1800 Bienville Drive, Monroe, LA 71201. USA \\ ${ }^{2}$ Department of Biology, College of Arts, Education and Science, University of Louisiana at Monroe, \\ 700 University Avenue, Monroe, LA 71209. USA \\ *Corresponding author E-mail: kjackson@ulm.edu
}

\begin{abstract}
Background: Metabolic syndrome is a collection of ailments resulting in a higher risk of cardiovascular disease and type II diabetes mellitus. It also results in prolonged endothelial dysfunction which promotes hypertension.

Objective: The current study examines the acute effect of carbon monoxide (CO) inhibition and nitric oxide (NO) stimulation in septal coronary arteries.

Methods: These studies were conducted in inactin anesthetized obese and lean Zucker rats (13-14 weeks of age). Coronary arteries were isolated from obese and lean Zucker rats and in vitro experiments were conducted. Isolated coronary arteries were pre-treated with chromium mesoporphyrin (CrMP) which is a heme oxygenase inhibitor and L-arginine, a NO precursor.

Results: Blood pressure, non-fasting blood glucose, HBCO, CO levels and Arginase I expression were higher in obese Zucker rats (ZR) as compared to the lean (L) group. Obese ZR had higher body, kidney and heart weights as compared to the LZR. Acetylcholine induced vasodilation was greatly attenuated in Obese ZR compared to the lean group. No differences in the diameters of the septal coronary artery were observed in both groups when treated with CrMP. However, pretreatment with L-arginine, abolished the differences between the groups.

Conclusion: This study demonstrates the potential of NO induction to improve coronary blood flow during metabolic syndrome induced endothelial dysfunction, where alterations in $\mathrm{CO}$ levels appeared to have no significant coronary effects.
\end{abstract}

Keywords: Metabolic Syndrome, Endothelial Dysfunction, Hypertension, L-Arginine, Endogenous CO.

\section{Introduction}

Metabolic syndrome continues to be a global problem with high prevalence in the United States. It is estimated that in USA about $40 \%$ of adults have metabolic syndrome by the time they reach 60 years of age (Ford et al. 2002, Ford et al. 2010). Metabolic syndrome contains a cluster of various risk factors which include atherogenic dyslipidemia, elevated blood pressure, endothelial dysfunction (Alessi \& Juhan 2008) and arterial stiffness (Stehouwer et al. 2008). Taking together these factors increase the risk of developing cardiovascular disease and type II diabetes mellitus.

Obese Zucker rats (ZR) are a mutant rat model, which replicates obese humans by sharing the symptoms of type II diabetes. Obese $\mathrm{ZR}$ is the result of a spontaneous mutation of autosomal recessive (fa) gene on chromosome 5. They have hyperphagia, insulin resistance (Kasiske et al. 1992), mild glucose intolerance, hyperlipidemia (Kaiske et al. 1992), hyperinsulinemia, moderate hypertension (Frisbee \& Stepp 2001, Durham \& Truett 2006, Shafrir \& Ziv 2009), early onset of obesity and metabolic syndrome along with mild hyperglycemia. They act as an excellent model of human obesity and type II diabetes which are both associated with hyperlipidemia and hypertension (Srinivasan \& Ramarao 2007).
$\mathrm{CO}$ is formed endogenously in the body by the enzymatic degradation of heme by the enzyme heme oxygenase (HO) (Jarmi \& Agarwal 2009) and other non-enzymatic degradations (Tenhunen et al. 1969). Metabolism of heme also results in the production of biliverdin which upon action of biliverdin reductase is converted to bilirubin (Tenhunen 1976). There are two major active isoforms of HO (i) the inducible HO-I and (ii) the constitutive HO-II (Maines 1997). Several stimuli such as hypoxia, oxidative stress, endotoxin, ischemia, hypertension and oxidants induce HO-I, which is otherwise expressed at low concentrations under normal conditions (Abraham \& Kappas 2005). Unlike HO-I, HO-II is constitutively expressed. Emerging evidence demonstrates the beneficial actions of $\mathrm{HO}$ on the cardiovascular system which includes attenuation of hypertension and the prevention of atherosclerosis and myocardial infarctions (Sabaawy et al. 2001, Juan et al. 2001, and Tang et al. 2005) as it was demonstrated that CO can relax vascular smooth muscles (Furchgott \& Jothianandan 1991, Stec et al. 2008). Previous studies demonstrated that increased CO formation from heme in obese ZR contributed to hypertension and endothelial dysfunction in resistance vessels (Durante \& Schafer 1998, Johnson et al. 2006). Therefore, we hypothesized that inhibition of $\mathrm{CO}$ restores coronary arterial endothelial function in obese ZR. 
The endothelium which is the interface between blood and the vessel wall plays a crucial role in vascular homeostasis. Endothelial cells produce potent vasoactive substances including nitric oxide (NO) and endothelin-1 which are vasodilatory and vasoconstrictor substances respectively (Perticone et al. 2001). The pathological condition resulting due to imbalance between vasodilatory and vasoconstrictive substances is characterized as endothelial dysfunction (Andreas et al. 2010). To date many cardiovascular abnormalities have been attributed to endothelial dysfunction. Endothelial dysfunction is a key factor promoting microvascular disease in resistance vessels, contributing to hypertension and metabolic syndrome (Johnson et al. 2006, Shatsila et al. 2013).

Alternatively, previous literature demonstrated that $\mathrm{CO}$ promotes endothelium dependent vasoconstriction by inhibiting endothelial NO formation (Johnson \& Johnson 2003) thus attenuating the vasodilatory effects of the nitric oxide system (White \& Marletta 1992, Thorup et al. 1999). Taken together, this suggests that CO and NO play crucial roles as regulators of normal vascular tone.

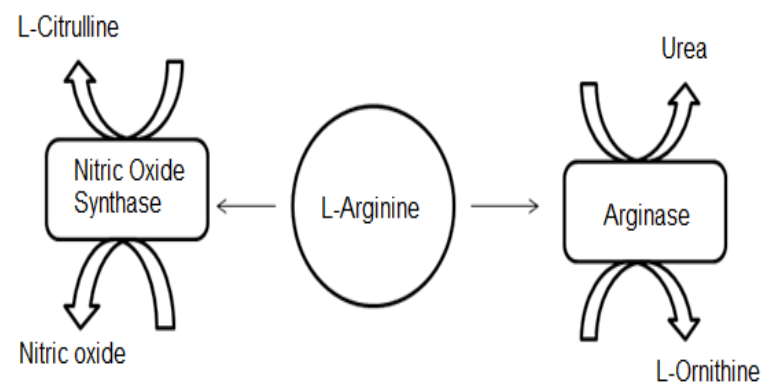

Fig. 1: L-Arginine, common substrate for both arginase and nitric oxide synthase. L-Arginine is converted to urea and L-ornithine by the arginase enzyme along with nitric oxide and L-citrulline by nitric oxide synthase.

NO acts as an important signaling molecule exhibiting a wide range of biological effects such as vasodilation, anti-platelet activity, anti-inflammatory actions, cardio-protection, and antihypertrophy (Strijdom et al. 2009). NO is endogenously biosynthesized by many cells from the amino acid L-arginine by the enzymatic action of nitric oxide synthase (NOS). NOS are significantly expressed in nervous and cardiovascular systems among a variety of tissues throughout the body. There is significant evidence which demonstrates that a decrease in bioavailability of $\mathrm{NO}$ results in various cardiovascular diseases and disorders (Vanhoutee \& Gao 2013, Sarkozy et al. 2013). The limiting factor for NO production is the availability of the substrate L-arginine. Previous studies have shown that arginase, which is an enzyme in the urea cycle, reduces NO production by competing with NOS which also has L-arginine as a substrate (Ming et al. 2004) (Fig. 1). Thus, we alternatively hypothesized that L-arginine would restore coronary arterial endothelial function in obese ZR.

\section{Methods}

\subsection{Chemicals}

Chromium mesoporphyrin (CrMP) was purchased from Frontier Scientific (Logan, UT, USA); Inactin (thiobutabarbital sodium), L-arginine and Acetylcholine (ACh) were obtained from Sigma (St. Louis, MO, USA).

\subsection{Animals}

Male lean $(\mathrm{Fa} / \mathrm{Fa}$ or $\mathrm{Fa} / \mathrm{fa}, \mathrm{n}=52)$ and obese $(\mathrm{fa} / \mathrm{fa}$, n=62) ZR (Harlan, Indianapolis, IN, USA) at 13 to 14 weeks of age were used for the experiments. Rats were housed in a controlled environment and had free access to a standard rodent diet (Harlan Teklad, Madison, WI, USA) and tap water. All procedures were approved by the University of Louisiana at Monroe Institutional Animal Care and Use Committee.

\subsection{Determination of Carboxyhemoglobin (HbCO) lev- els}

Small blood samples $(0.05 \mathrm{ml})$ were drawn from obese and lean ZR. Blood was analyzed on a $\mathrm{pH} / \mathrm{blood}$ gas \& electrolyte analyzer for carboxyhemoglobin concentration (Bayer Rapid Lab 865, Diamond Diagnostic, Holiston, MA, USA).

\subsection{Determination of $\mathrm{CO}$ excretion}

Matched awake lean and obese ZR were placed in an acrylic airtight chamber with the outflow leading to a solid phase gas chromatography unit (custom design, Peak, Mountain view, CA,USA) for the determination of endogenous $\mathrm{CO}$ concentration as previously described (Vreman et al. 2009, Jackson et al. 2011) . Briefly, the chamber was continuously purged with purified air. Outflow was sampled at 2 minutes intervals and the average of four measurements was used to calculate the $\mathrm{CO}$ excretion rate for the whole animal after a $30 \mathrm{~min}$ equilibration period. This is an established model for measuring CO production (Johnson et al. 2006, Vreman et al. 2005).

\subsection{Blood pressure measurements}

Rats were anesthetized with a single injection of thiobutabarbital sodium $(120 \mathrm{mg} / \mathrm{kg}$ ip) and a tracheal tube was inserted to maintain an open airway. A catheter (PE-50 tubing filled with heparinized saline) was implanted into a carotid artery to measure mean arterial pressure (MAP)/heart rate (HR). The arterial catheter was connected to a pressure transducer (model TSD104A, Biopac Systems, Santa Barbara, CA, USA) and MAP/HR was recorded on a computer.

\subsection{Measurement of diameter in isolated septal coro- nary artery}

The septal coronary artery was isolated, removed and placed into a dish containing ice-cold Krebs buffer. It was allowed to equilibrate for $15 \mathrm{~min}$. It was then transferred to a water-jacketed vessel chamber containing Krebs buffer at room temperature and two glass micropipettes filled with Krebs buffer. After the artery was mounted on the proximal micropipette and secured with a ligature, the lumen was flushed to remove residual blood and the other end of the artery was mounted on the distal micropipette (Sun et al. 1994). For internal diameter measurements, the vessel chamber was mounted on a stage of an inverted microscope (Nikon TS 100 F) fitted with a CCD video camera. Silicone tubing was used to connect the proximal micropipette to a pressure servocontroller (model/CH/200/Q, Living systems instrumentation, Burlington, VT, USA) and the distal pipette to a stopclock. Continuous nonrecirculating superfusion with Krebs buffer, bubbled with 95\% $\mathrm{O} 2-5 \% \mathrm{CO}_{2}$ was then started while the lumen was perfused. After several minutes of perfusion, the distal stopcock was closed and the intraluminal pressure was allowed to increase to $80 \mathrm{~mm} \mathrm{Hg}$ (Kozma et al. 1999). After a 60 min stabilization period, the HO inhibitor, CrMP, the NOS substrate, L-Arginine or vehicle was included in the superfusion buffer, $20 \mathrm{~min}$ before the experiment. This treatment regime was continued throughout the experiment. After the pre-treatment period, increasing concentration of the endothelium dependent vasodilator ACh was added to the superfusion buffer. Each concentration was tested for $5 \mathrm{~min}$; internal diameter was recorded every minute, and the average of the last two measurements was used to determine the response.

\subsection{Plasma nitrite levels}

Plasma nitrite levels were measured using a colorimetric assay kit (Cayman chemicals, Ann Arbor, MI, USA). 


\subsection{Western blot analysis}

The tissue homogenates were assayed for arginase I protein by western blot analysis as previously described (Berkowitz et al 2003).

\subsection{Statistics}

Data were expressed as mean \pm SE. Data were analyzed by repeated measure analysis of variance (ANOVA) followed by orthogonal contrast when appropriate (Sigma stat 3.0). P < 0.05 was accepted as statistically significant. Bonferroni correction was employed in the final analysis of completed series $(\alpha=0.05)$.

Table 1: Various metabolic parameters: Mean Arterial Pressure (MAP), Heart rate, carboxyhemoglobin and blood glucose of obese Zucker rats were higher than lean $\mathrm{ZR}(* \mathrm{P}<0.05)$. Values are expressed as mean $\pm \mathrm{SE}$.

\begin{tabular}{lll}
\hline Variable & Lean Zucker Rat & Obese Zucker Rat \\
\hline MAP $(\mathrm{mmHg})$ & $121 \pm 4$ & $154 \pm 3^{*}$ \\
Heart Rate $(\mathrm{bpm})$ & $353 \pm 10$ & $386 \pm 7$ \\
HbCO\% & $3.0 \pm 0.1$ & $3.9 \pm 0.1^{*}$ \\
Blood Glucose $(\mathrm{mg} / \mathrm{dL})$ & $140 \pm 3$ & $186 \pm 7^{*}$ \\
\hline
\end{tabular}

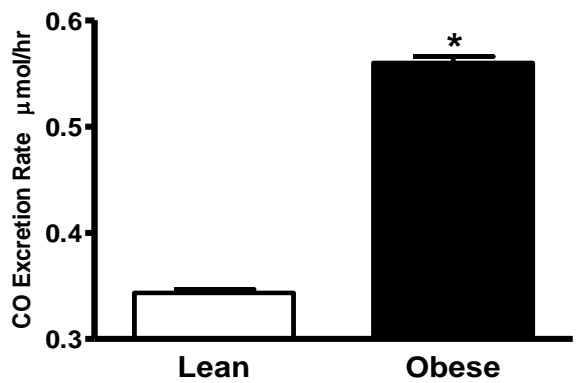

Fig. 2: Respiratory Carbon monoxide excretion: Expired carbon monoxide was measured in obese and lean ZR. It was higher in awake obese ZR compared to the lean group $(* \mathrm{P}<0.05)$. Values are expressed as mean $\pm \mathrm{SE}$.

\section{Results}

\subsection{Blood pressure and CO excretion with glucose measurements}

Obese ZR had elevated blood pressures, HbCO (Table 1) and endogenous CO levels (Fig. 2) as compared to lean ZR. Non fasting blood glucose was higher in obese ZR compared to lean ZR (Table 1).

\subsection{Body and organ weight measurement}

Table 2 summarizes the body, kidney and heart weights of lean and obese ZR. Obese ZR had higher body weights, as well as kidney and heart weights, when compared with lean ZR. The characteristics of elevated blood pressure and increased body weight demonstrate that obese ZR is an excellent model to represent obesity and hypertension.

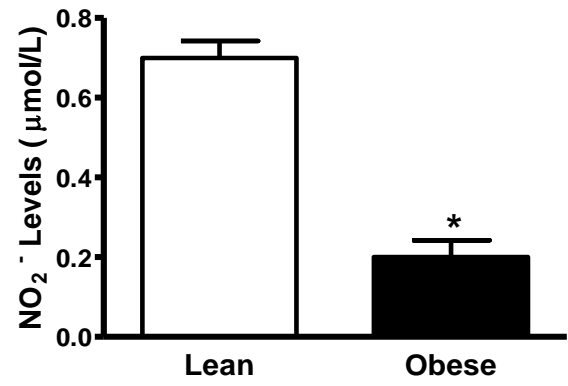

Fig. 3: Plasma nitrite levels: Nitrites are the breakdown products of nitric oxide. The nitrite levels in plasma are decreased in obese $\mathrm{ZR}(* \mathrm{P}<0.05)$ Values are expressed as mean $\pm \mathrm{SE}$
Table 2: Physical Measurements of Lean and Obese ZR: Body, kidney and heart weights were higher in obese ZR as compared to lean ZR $(* \mathrm{P}<0.05)$. Values are expressed as mean $\pm \mathrm{SE}$.

\begin{tabular}{lll}
\hline Variable & Lean Zucker Rat & Obese Zucker Rat \\
\hline Body Weight $(\mathrm{g})$ & $309 \pm 14$ & $551 \pm 13^{*}$ \\
Kidney Weight $(\mathrm{g})$ & $1.03 \pm 0.04$ & $1.38 \pm 0.04^{*}$ \\
Heart Weight $(\mathrm{g})$ & $0.87 \pm 0.08$ & $1.15 \pm 0.04^{*}$ \\
\hline
\end{tabular}

\subsection{Plasma nitrite levels}

There was a significant decrease in plasma nitrite levels in obese ZR as compared to lean ZR (Fig. 3)

\subsection{Isolated pressurized septal coronary artery meas- urements}

The septal coronary artery was isolated from obese and lean ZR. Obese ZR arteries had larger internal diameters. An endotheliumdependent vasodilator, $\mathrm{ACh}$, promoted concentration-dependant vasodilation in arteries isolated from lean and obese ZR (Fig. 4). $\mathrm{ACh}$ induced vasodilation was greatly attenuated in obese $\mathrm{ZR}$ compared to the lean group (Fig. 4). Twenty minute pretreatment with the HO inhibitor, CrMP (Fig. 5), promoted no significant difference in diameters compared to that of vehicle (Fig. 4) in both groups.

Change in Diameter Vehicle

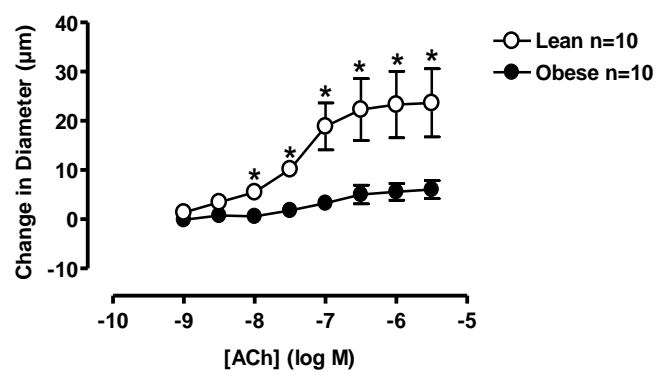

Fig. 4: Vessel Diameter: Concentration dependent change in internal diameter of septal coronary arteries in response to the endothelium dependent vasodilator, ACh. ACh induced vasodilation was greatly attenuated in obese ZR compared with the lean group $(* \mathrm{P}<0.05)$. Values are expressed as mean $\pm \mathrm{SE}$.

\subsection{Aortic Arginase I expression and L-arginine pre- treatment}

The negative results from the CrMP studies lead to us to examine the alternate hypothesis that differences in endothelial function in obese ZR and lean ZR could be produced by elevations in arginase, which promotes a decrease in NO availability via a decrease in L-Arginine substrate (Fig. 1). From the western blot analysis, there was approximately a two fold increase in Arginase I expression in obese ZR than in lean ZR (Fig. 6). Acute in vitro pretreatment with L-arginine, common substrate for NOS and arginase (Fig. 1), enhanced the maximal responses in obese ZR arteries as compared to vehicle treatment (Fig. 7). Furthermore, acute in vitro pretreatment with L-arginine abolished the difference between the lean and obese groups (Fig. 8).

\section{Discussion}

It was previously documented that obese ZR have increased endogenous $\mathrm{CO}$ production, resulting in endothelial dysfunction and hypertension, which ultimately promotes metabolic syndrome (Johnson et al. 2006). Similarly, in the present study, we observed some of the common characteristics of metabolic syndrome namely, increased body weight, elevated blood glucose and hypertension. 
Emerging evidence demonstrates that increased HO-derived $\mathrm{CO}$ production may result in hypertension in obese ZR. It was previously reported that, in isolated gracilis muscle arterioles, exogenously and endogenously formed $\mathrm{CO}$ promoted endotheliumdependent vasoconstriction which attenuated NO mediated vasodilation leading to hypertension (Johnson \& Johnson 2003). Given the established role of $\mathrm{CO}$ in peripheral arteries, the present study focused on the role of $\mathrm{CO}$ in the cardiovascular system wherein we tested the hypothesis that inhibition of $\mathrm{CO}$ restores coronary

\section{Change in Diameter CrMP}

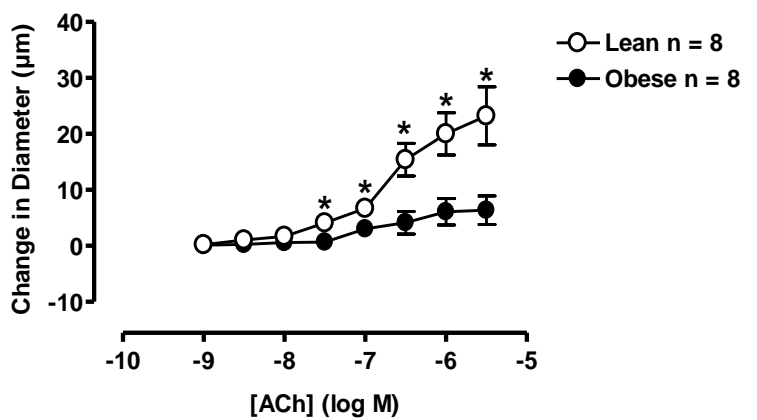

Fig. 5: Vessel Diameter: Concentration dependent change in internal diameter of septal coronary arteries in response to endothelium dependent vasodilator, ACh during pretreatment with an inhibitor of heme oxygenase, chromium mesoporphyrin (CrMP). ACh induced vasodilation was greatly attenuated in obese ZR compared with lean group. No significant difference was observed in CrMP treated and vehicle treated groups $(* \mathrm{P}<0.05)$. Values are expressed as mean $\pm \mathrm{SE}$.

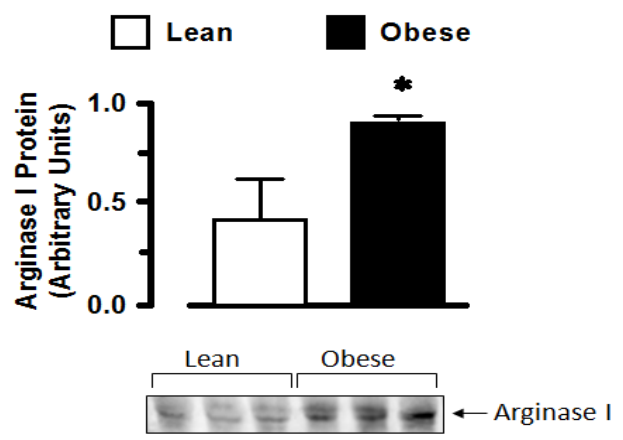

Fig. 6: Arginase I expression: Western blot analysis of Arginase 1 expression in coronary arteries. A significant increase in aortic arginase levels was observed in obese ZR compared to lean $\mathrm{ZR}(* \mathrm{P}<0.05)$. Values are expressed as mean $\pm \mathrm{SE}$.

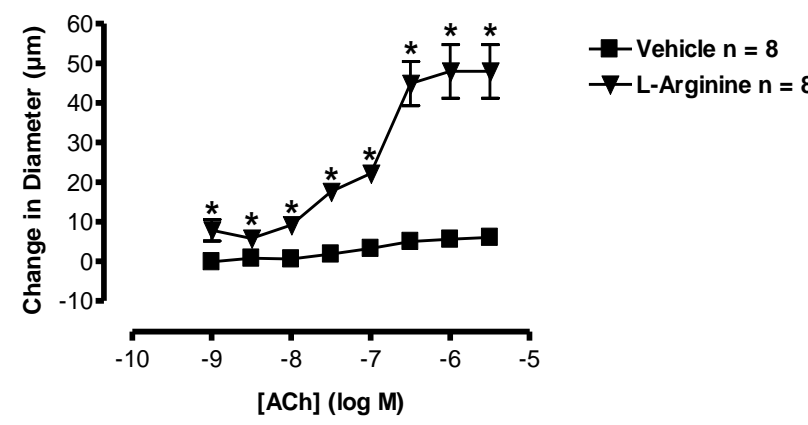

Fig. 7: Vessel Diameter: Concentration dependent change in internal diameter of septal coronary arteries in response to endothelium dependent vasodilator, ACh and when treated with vehicle and L-arginine, substrate for nitric oxide in obese ZR. Vasodilation was observed in L-arginine treated group compared to vehicle treated $(* \mathrm{P}<0.05)$. Values are expressed as mean $\pm \mathrm{SE}$. arterial endothelial function in obese ZR. However in the present study, there were no significant changes in dilation of septal coronary arteries when treated with CrMP compared to vehicle. Thus it appears that the role of $\mathrm{CO}$ in controlling vascular tone is more prominent in periphery arteries than in the coronary arteries.

NO is an important mediator of endothelium dependent vasodilation, therefore NO plays a significant role in regulating vascular tone. It was observed that obese ZR have lower nitrite levels indicating the presence of lower nitric oxide concentration. This was in turn supported by the presence of higher arginase-I concentrations in obese ZR. As previously described, arginase reduces NO production from L-arginine by competing with NOS. Thus, suggesting that there is a decrease in NO bioavailability due to an increase in arginase competition for NOS.

Previously it was reported that low concentrations of CO promote vasodilation, on the other hand at physiological concentrations $\mathrm{CO}$ inhibited NOS in isolated renal resistance vessels (Johnson \& Johnson 2003). It was also previously documented that, in isolated gracilis muscle arterioles, CO-induced vasoconstriction was abolished by L-arginine pretreatment suggesting the protective role of L-arginine. However, to examine the prominent role of $\mathrm{NO}$ in coronary vessels, we proposed an alternative hypothesis that Larginine restores coronary arterial endothelial function in obese ZR.

L-arginine is a semi essential amino acid, which plays a prominent role in numerous physiological processes. L-arginine acts as a common substrate for arginase as well as for NOS, which produces the signaling molecule NO. NO plays a significant role in various physiological processes which include decreasing vascular tone, endothelial cell death, extracellular matrix deposition, smooth muscle cell proliferation, platelet and leucocyte activation. NO deficiency results in various pathological disorders including hypertension, atherosclerosis, diabetes and ischemia-reperfusion injury (Durante et al. 2007). Various clinical studies suggested that L-arginine exhibits endocrine actions in regulating insulin sensitivity and vasodilator function. It was previously documented that in vivo L-arginine supplementation, enhances endothelial NOS (Kohi et al. 2004).

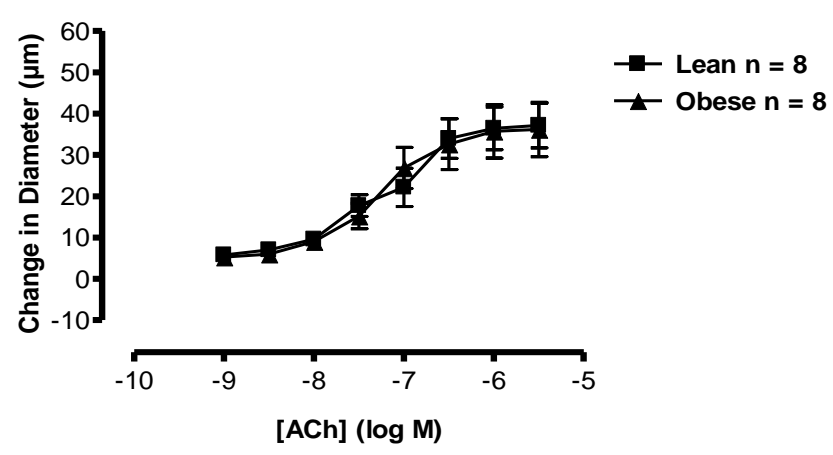

Fig. 8: Vessel Diameter: Concentration dependent change in internal diameter of septal coronary arteries in response to endothelium dependent vasodilator, ACh during pretreatment with L-arginine, substrate for NOS. This treatment with $\mathrm{L}$-arginine abolished the differences between lean and obese ZR.

In the present study, acute administration of L-arginine to isolated coronary arteries produced an increase in NO levels and abolished the vasodilatory differences between obese and lean ZR. In addition, long term administration of L-arginine also had a favorable effect on the endothelium (Cylwik et al. 2005).

In summary, we conclude that the coronary endothelial dysfunction observed in obese ZR could not be abolished via inhibition of HO. Consequently, L-arginine treatment could abolish the difference between lean and obese ZR. Thus presenting a chief role for 
NO in maintaining the vascular tone in the cardiovascular system unlike the periphery where $\mathrm{CO}$ appears to have a major role.

\section{Acknowledgements}

The authors would like to sincerely thank Dr. Robert A. Johnson and Dr. Fruzsina K. Johnson for their assistance with the completion of this manuscript and for use of their laboratory equipment. This study was supported by research grants from LEQSF (200912)-RD-A-22.

\section{References}

[1] Ford ES, Giles WH, \& Dietz WH (2002) Prevalence of the metabolic syndrome among US adults: findings from the third National Health and Nutrition Examination Survey. JAMA 287, 356-359.

[2] Ford ES, Li C, \& Zhao G (2010) Prevalence and correlates of metabolic syndrome based on a harmonious definition among adults in the US. J Diabetes 2, 180-193.

[3] Alessi MC \& Juhan (2008) Vague I: Metabolic syndrome, haemostasis and thrombosis. Thromb Haemost 99, 995-1000.

[4] Stehouwer CD, Henry RM, \& Ferreira I (2008) Arterial stiffness in diabetes and the metabolic syndrome: a pathway to cardiovascular disease. Diabetologia 51, 527-539.

[5] Kasiske BL, O Donnell MP, \& Keane WF (1992) The Zucker ra model of obesity, insulin resistance, hyperlipidemia and renal injury. Hypertension 19, 110-115.

[6] Frisbee JC \& Stepp DW (2001) Impaired NO-dependant dilation of skeletal muscle arterioles in hypertensive obese Zucker rats. Am J Physiol Heart Circ Physiol 281, H1304-H1311.

[7] Durham HA \& Truett GE (2006) Development of insulin resistance and hyperphagia in Zucker fatty rats. Am J Physiol Regul Interg Comp Physiol 210, R652-658.

[8] Shafrir E \& Ziv E (2009) A useful list of spontaneously arising animal models of obesity and diabetes. Am J Physiol Endocrinol Metab 296, E1450-E1452.

[9] Srinivasan K \& Ramarao P (2007) Animal models in type 2 diabetes research: an overview. Indian J Med Res 125, 451-472.

[10]Jarmi T \& Agarwal A (2009) Heme oxygenase and renal disease. Curr Hypertens Rep 11, 56-62.

[11]Tenhunen R, Marver HS \& Schmid R (1969) Microsomal heme oxygenase. Characterization of the enzyme. J Biol Chem 244, 63886394.

[12]Tenhunen R (1976) The enzymatic conversion of heme to bilirubin in vivo. Ann Clin Res 8, 2-9.

[13]Maines MD (1997) The heme oxygenase system: a regulator of second messenger gases. Annu Rev Pharmacol Toxicol 37, 517-554.

[14]Abraham NG \& Kappas A (2005) Heme oxygenase and the cardiovascular-renal system. Free Radic Biol Med 39, 1-25.

[15] Sabaawy HE, Zhang F, Nguyen X, Elhosseiny A, Nasjletti A, Schwartzman M, Dennery P, Kappas A \& Abraham NG (2001) Human heme oxygenase-1 gene transfer lowers blood pressure and promotes growth in spontaneously hypertensive rats. Hypertension 38, 210-215.

[16]Juan SH, Lee TS, Tseng KW, Liou JY, Shyue SK, Wu K \& Chau LY (2001) Adenovirus-mediated heme oxygenase-1 gene transfer inhibits the development of atherosclerosis in apolipoprotein E-deficient mice. Circulation 104, 1519-1525.

[17]Tang YL, Qian K, Zhang YC, Shen L \& Phillips MI (2005) A vigilant, hypoxia- regulated heme oxygenase-1 gene vector in the heart limits cardiac injury after ischemia-reperfusion in vivo. $J$ Cardiovasc Pharmacol Ther 10, 251-263.

[18]Furchgott RF \& Jothianandan D (1991) Endothelium-dependent and independent vasodilation involving cyclic GMP: relaxation induced by nitric oxide, carbon monoxide and light. Blood Vessels 28, 52-61.

[19] Stec DE, Drummond HA, \& Vera T (2008) Role of carbon monoxide in blood pressure regulation. Hypertension 51, 597-604.

[20]Durante W \& Schafer Al (1998) Carbon monoxide and vascular cell function (review). Int J Mol Med 2, 255-262.

[21]Johnson FK, Johnson RA, Durante W, Jackson KE, Stevenson BK, \& Peyton KJ (2006) Metabolic syndrome increases endogenous carbon monoxide production to promote hypertension and endothelial dysfunction in obese Zucker rats. Am J Physiol Regul Integr Comp Physiol 290, R601-608.
[22]Perticone F, Ceravolo R, Pujia A, Ventura G, Iacopino S, Scozzafava A, Ferraro A, Chello M, Mastroroberto P, Verdecchia P, \& Schillaci G (2001) Prognostic significance of endothelial dysfunction in hypertensive patients. Circulation 104, 191-196.

[23] Andreas J, Flammer T, \& Luscher F (2010) Three decades of endothelium research: from the detection of nitric oxide to the everyday implementation of endothelial function measurements in cardiovascular diseases. Swiss Medical Weekly 140, w13122.

[24] Shantsila E, Shantsila A, Blann AD, \& Lip GY (2013) Left Ventricular Fibrosis in Atrial Fibrillation. Am J Cardiol 111, 9961001 .

[25]Johnson FK \& Johnson RA (2003) Carbon monoxide promotes endothelium-dependent constriction of isolated gracilis muscle arterioles. Am J Physiol Regul Integr Comp Physiol 285, R536-541.

[26] White KA \& Marletta MA (1992) Nitric oxide synthase is a cytochrome P-450 type hemoprotein. Biochemistry 31, 6627-6631.

[27]Thorup C, Jones CL, Gross SS, Moore LC, \& Goligorsky MS (1992) Carbon monoxide induces vasodilation and nitric oxide release but suppresses endothelial NOS. Am J Physiol 277, F882-889.

[28] Strijdom H, Chamane N, \& Lochner A (2009) Nitric oxide in the cardiovascular system: a simple molecule with complex actions. Cardiovasc J Afr 20, 303-310

[29]Vanhoutte PM \& Gao Y (2013) Beta blockers, nitric oxide, and cardiovascular disease. Curr Opin Pharmacol 13, 265-273.

[30]Sárközy M, Zvara A, Gyémánt N, Fekete V, Kocsis GF, Pipis J, Sz CG, Csonka C, Puskás LG, Ferdinandy P, \& Csont T (2013) Metabolic syndrome influences cardiac gene expression pattern at the transcript level in male ZDF rats. Cardiovasc Diabetol 12, 16-32.

[31]Ming XF, Barandier C, Viswambharan H, Kwak BR, Mach F, Mazzolai L, Hayoz D, Rusconi S, Montani JP, \& Yang Z (2004) Thrombin stimulates human endothelial arginase enzymatic activity via RhoA/ROCK pathway: implications for atherosclerotic endothelial dysfunction. Circulation 110, 3708-3714.

[32] Vreman HJ, Knauer Y, Wong RJ, Chan ML, \& Stevenson DK (2009) Dermal carbon monoxide excretion in neonatal rats during light exposure. Pediatr Res 66, 66-69.

[33]Jackson KE, Jackson DW, Quadri S, Reitzell MJ, \& Navar LG (2011) Inhibition of heme oxygenase augments tubular sodium reabsorption. Am J Physiol Renal Physiol 300, F941-F946.

[34]Vreman HJ, Wong RJ, Kadotani T, \& Stevenson DK (2005) Determination of carbon monoxide (CO) in rodent tissue: effect of heme administration and environmental $\mathrm{CO}$ exposure. Anal Biochem $341,280-289$

[35] Sun D, Kaley G, \& Koller A (1994) Characteristics and origin of myogenic response in isolated gracilis muscle arterioles. Am J Physiol 266, H1177-1183.

[36] Kozma F, Johnson RA, Zhang F, Yu C, Tong X, \& Nasjletti A (1999) Contribution of endogenous carbon monoxide to regulation of diameter in resistance vessels. Am J Physiol 276, R1087-1094.

[37]Berkowitz DE, White R, Li D, Mihas KM, Cernetich A, Kim S, Burke S, Shoukas AA, Nyhan D, Champion HC, \& Hare JM (2003) Arginase reciprocally regulates nitric oxide synthase activity and contributes to endothelial dysfunction in aging blood vessels. Circulation 108, 2000-2006

[38]Durante W, Johnson FK, \& Johnson RA (2007) Arginase: a critical regulator of nitric oxide synthesis and vascular function. Clin Exp Pharmacol Physiol 34, 906-911.

[39]Kohli R, Meininger CJ, Haynes TE, Yan W, Self JT, \& Wu G (2004) Dietary L-arginine supplementation enhances endothelial nitric oxide synthesis in streptozotocin-induced diabetic rats. J Nutr 134, 600-608.

[40]Cylwik D, Mogielnicki A, \& Buczko W (2005) L-arginine and cardiovascular system. Pharmacol Rep 57, 14-22. 